\title{
New Worlds Observer Mission Design
}

\author{
Tiffany Glassman, Amy Lo, Chuck Lillie, and Keith Kroening \\ Northrop Grumman Corporation, Redondo Beach, CA, 90278
}

\begin{abstract}
New Worlds Observer is an external starshade mission designed to observe Earth-like planets around nearby stars. A telescope spacecraft operates in the "shadow" cast by a starshade spacecraft, located tens of thousands of kilometers away. We explore the requirements that these two free-flying spacecraft place on the orbital, propulsion, control, and communication systems and some baseline solutions for each. This concept is young and we are still learning about how the system operates. However, we have developed a strawman design that meets all of the NWO mission requirements.
\end{abstract}

\section{Introduction}

New Worlds Observer (NWO) is a mission concept designed to identify extra-solar planets. NWO consists of a telescope and an external starshade aligned with a target star. A sketch of the NWO architecture is shown in Fig. 1.

\section{Exoplanet}

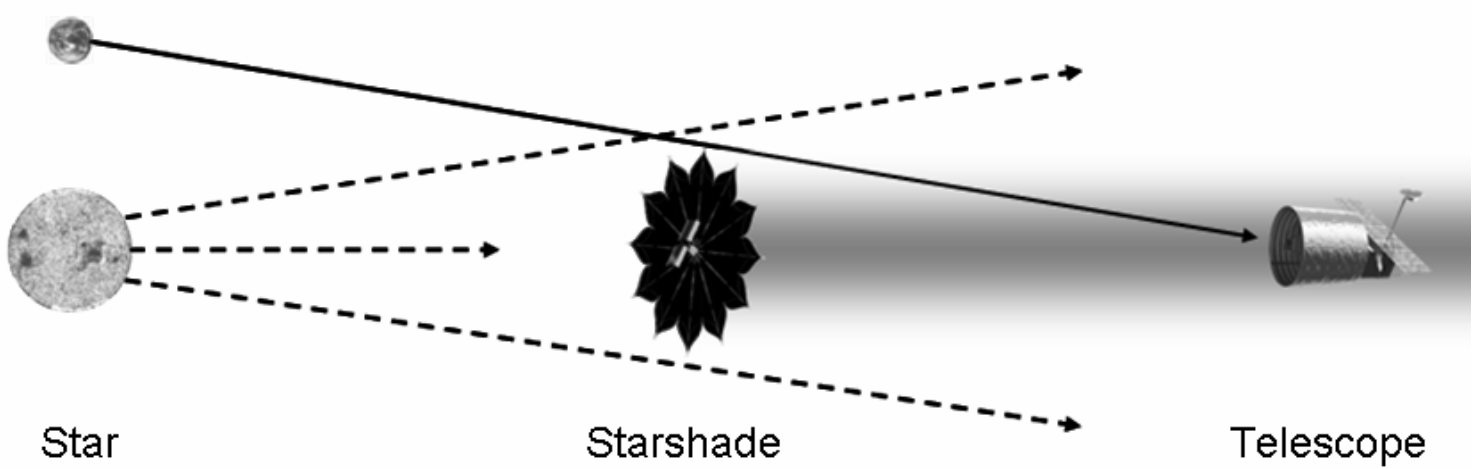

Figure 1. The basic architecture of the NWO mission. A generic telescope is used in conjunction with the NWO starshade to block out the on-axis starlight and detect and characterize an off-axis source the extra-solar planet.

The problem of directly detecting planets around other stars requires very high contrast at very small angles to the star. The starshade is capable of creating a given contrast ratio at all angles greater than some minimum, called the Inner Working Angle (IWA). The requirements for the telescope are only that it can detect a faint point source and that it has the resolution necessary to differentiate between two objects very close together. All capabilities aimed specifically at planet detection are produced by the design of the starshade.

In a recent paper, Ref. 1 showed that a starshade with an offset hypergaussian transmission profile provides a deep, broadband null for on-axis light and high transmission for an off-axis source. This starshade is at the heart of the NWO architecture and is the starshade design discussed in this paper.

The offset hypergaussian apodization function is given by:

$$
A(\rho)=0 \quad \rho<a
$$




$$
A(\rho)=1-\exp \left[-\left(\frac{\rho-a}{b}\right)^{n}\right] \quad \rho \geq a
$$

where $a$ is the radius of a central region of zero transmission, $b$ is the fall-off radius of the exponential function, $n$ is the index that determines how quickly the hypergaussian function falls with radius, and $\rho$ is the starshade radius. The starshade is a binary mask with flower-like petals that are completely opaque. Each petal provides a fraction, $T(\rho)$, of the total transmission of the mask:

$$
T(\rho)=\frac{2 \pi}{P}[1-A(\rho)]
$$

where $P$ is the number of petals. The 1-dimensional apodization function and the 2-dimensional, binary starshade are shown in Fig. 2.
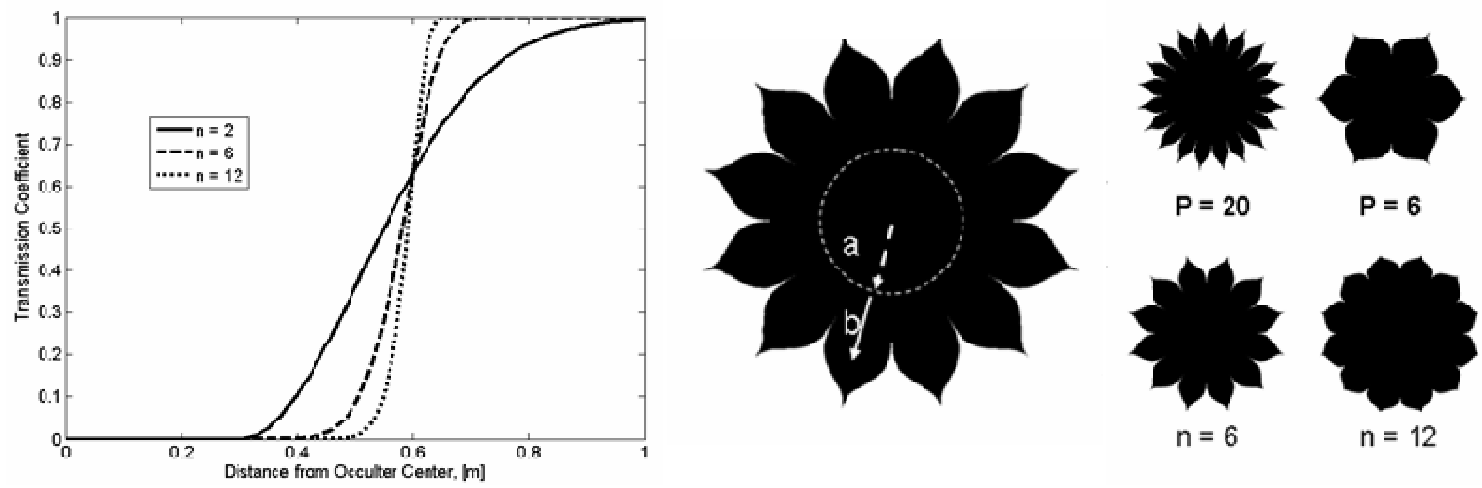

Figure 2. Left: The apodization function, $A(\rho)$, for the NWO starshade, showing several different hypergaussian functions. Center: A 12-petaled, binary starshade with $n=6$. Right: Illustrations of starshade shapes with different petal numbers and values of $\mathbf{n}$.

The NWO architecture is very flexible ${ }^{2}$. Depending on the performance that is required and the size of mission that can be afforded, we would choose the starshade, telescope size, and starshade-telescope distance. In this paper we will focus on one example, though all the conclusions are valid for other mission scales. We will look at a $50 \mathrm{~m}$ diameter starshade and a $4 \mathrm{~m}$ telescope, operating 80,000 $\mathrm{km}$ apart. The mass of the starshade for this case would be about $3000 \mathrm{~kg}$. This setup would be able to suppress the starlight by a factor of $10^{-10}$ with an IWA of $\sim 50$ mas. This system would be able to find and characterize extra-solar terrestrial planets.

In this paper, we will focus on the orbital and alignment issues of operating these two, widely separated spacecraft. We will discuss the best orbit to place them in and the ability to realign the system to look from one target star to the next. We will cover the ability to keep the two spacecraft aligned during a science observation. We will discuss the control loops required for both of these stages, the communications design that they drive, and the propulsion systems that will be needed, and the amount of fuel used.

\section{Orbit}

The fundamental requirement for the NWO orbit is that the system must be located in an orbit so that it can observe a sufficient number of target stars over the mission lifetime to detect a high number of terrestrial planets. Terrestrial planets shine by reflected starlight, and this means that they are usually very dim. If we looked at the solar system from 10 parsecs away, the Earth would be a $30^{\text {th }}$ magnitude object. For a 4 meter telescope, the photon arrival rate from a $30^{\text {th }}$ magnitude object is on the order of a few photons per second. This means that to get a significant detection, the orbit of the NWO system must allow the telescope, the starshade, and the target star to remained aligned for hours, or even days. 
Due to the uncertainties in the number of terrestrial planets around nearby stars, and the lack of currently identified targets, NWO must be able to conduct a survey of a sufficiently large number of stars in order to have a high probability of finding a few terrestrial planets. For example, if the goal of NWO is to detect 20 terrestrial planets, and $50 \%$ of all planetary systems have a terrestrial planet, then the minimum number of targets that need to be surveyed is 40 . However, the terrestrial planet will not always be visible to the telescope; if we are looking in the plane of the solar system, the Earth will be in the right orientation to be seen during by this system during approximately a third of its orbit. Therefore, a crude measurement of the total number of target stars that need to be visited is $~ 120$. The ideal candidate stars are spread widely in the sky, so the NWO orbit must allow a large sky coverage.

In visiting these 120 systems, we will need to go back and perform follow-up observations of the same target star, known as revisits, in order to pin down the orbital parameters. At a minimum 4 visits, separated by a significant fraction of the orbital period, are necessary to establish an estimate of the orbit and determine whether the target is a terrestrial planet. NWO's orbit must allow for sufficient and flexible revisits.

In summary, we have derived three top level requirements for the NWO orbit:

1) it must allow NWO to be maintain alignment with a target star for hours or even days

2) it must have full, or close to full, sky coverage

3) it must be able to do a large number (e.g. 100+) of observations

4) it must allow repeat alignments at an appropriate frequency.

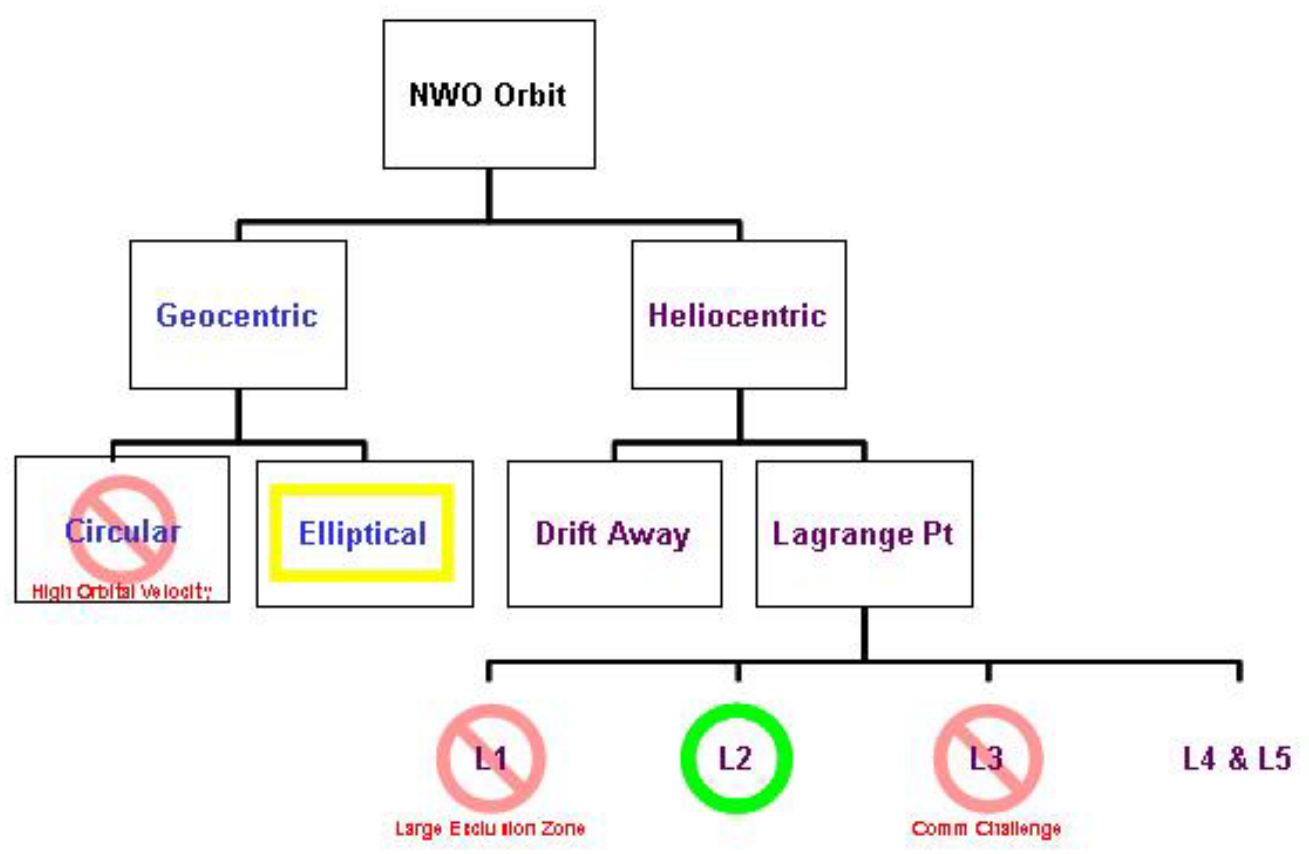

Figure 3: The trade space of possible orbits for the NWO mission. Our current baseline orbit is an L2 halo orbit, though others remain in the trade space.

Candidate orbits for NWO can be either geocentric or heliocentric. We can rule out most geocentric orbits, particularly circular orbits, from LEO to GEO. These orbits have such high orbital velocities that it would be very difficult to line up the starshade and the telescope with a target star for a sufficient period of time using a reasonable amount of fuel. A very elliptical (Molniya-like) orbit may offer enough integration time to enable limited observations. However, the sky coverage would be low. In general, geocentric orbits are not optimal for NWO.

Heliocentric orbits present the more attractive alternative and can be classified in two categories: driftaway and Lagrange-point orbits. The trade space for these orbit choices is presented in Fig. 3 and we discuss the pros and cons of each orbit below. 


\section{A. Drift-Away Orbits}

An Earth drift-away orbit has the advantage of putting NWO in a benign environment, but has a long transit time if NWO is to be placed far enough away so that Earthshine does not cause reflections on the surface of the starshade. This orbit uses minimum fuel for retargeting and stationkeeping, as the gravitational perturbations on the two spacecraft are very similar. These orbits remain in the trade space.

\section{B. Lagrange Points}

The Lagrange point orbits are very useful since minimum fuel needs to be used to maintain the orbit and the spacecraft remain a constant distance away from Earth. There are four Lagrange points of interest: L1, L2, L3, and L4/L5.

L1 is not an ideal location for NWO, since the Sun and the Earth are $180^{\circ}$ apart in the sky, creating two exclusion zones which significantly narrow the sky coverage. This is excluded from the trade space.

L2 is the current baseline location for NWO - while it has a long transit time, it enables the maximum amount of viewable sky at one time. Orbits about L2 have a 6 month period, which means the same patch of sky may be viewed up to 4 times a year, significantly increasing the flexibility of the revisits.

L3 has the same orbital properties as L2, but is on the other side of the Sun and therefore poses significant communication challenges. This is excluded from the trade space.

L4 \& L5 orbits have a long transit time and have the disadvantage of being farther away than L2, which increases the communication requirements. They will also have limits on the available sky coverage due to separate Earth- and Sun-exclusion zones. However, preliminary calculations have shown that movements at these Earth trailing (or leading) orbits require very little fuel. Therefore, they remain in the trade space.

\section{Fuel Usage}

\section{A. Retargeting}

The most fuel-expensive part of the NWO mission is moving the starshade from one target star to the next, which we call "retargeting". The large separation of the starshade from the telescope means the starshade must travel thousands of kilometers between targets. Since the mission requires a large sky coverage, careful scheduling of the targets is necessary. Using an L2 halo orbit as the baseline, we present some of the considerations of scheduling such a mission and sample fuel usage for retargeting a 50 meter starshade operated at $80,000 \mathrm{~km}$ from the telescope.

One of the top level parameters in creating the retargeting schedule is the target star sample. By including more stars in the target list, we decrease the average separation between the targets. For a sample of only 50 stars, evenly distributed in the sky, the average separation is $30^{\circ}$. If we include 250 stars in the target list, the average separation is $15^{\circ}$; if we include 1000 stars, the separation is $7^{\circ}$. This needs to be balanced against a scientifically culled list of ideal stellar candidates that may harbor planets. We currently have very little data on what such an ideal star would be; therefore, the baseline is to use nearby stars that are Sun-like. There is the potential that by the time NWO launches, previous ground- and space-based observations will have gathered more information about the potential target systems, simplifying the scheduling process.

The amount of fuel needed to do a given retargeting maneuver is a function of not just the size of the slew and the mass of the starshade, but also how long we allow for the slews to proceed. For example, for a starshade-telescope distance of $80,000 \mathrm{~km}$ and a total starshade mass of $3000 \mathrm{~kg}$, the amount of $\Delta \mathrm{v}$ to do a $15^{\circ}$ slew varies from $\sim 110 \mathrm{~m} / \mathrm{s}$ if we do this slew as fast as possible (about 9 days) to $\sim 40 \mathrm{~m} / \mathrm{s}$ if we do this slew in 13 days. A good average would be to take 10 days to do a $15^{\circ}$ slew which uses $\sim 60 \mathrm{~m} / \mathrm{s}$ of $\Delta \mathrm{v}$. For a 5 year mission, this would allow us to make $\sim 180$ observations using a total of $\sim 11,000 \mathrm{~m} / \mathrm{s}$ of $\Delta \mathrm{v}$. If the average distance between targets is $30^{\circ}$, the optimal time to do this slew is $\sim 14$ days (for the same starshade-telescope distance and starshade mass). This would use $\sim 90 \mathrm{~m} / \mathrm{s}$ of $\Delta \mathrm{v}$ per target, allowing us to observe $\sim 130$ targets in 5 years using a total of $\sim 12,000 \mathrm{~m} / \mathrm{s}$ of $\Delta \mathrm{v}$.

Due to the large $\Delta \mathrm{v}$ required, a chemical propulsion system would require carrying unreasonable amounts of fuel. We would need to carry $15,000 \mathrm{~kg}$ of hydrazine to produce the $11,000 \mathrm{~m} / \mathrm{s}$ of $\Delta \mathrm{v}$. If we used Solar Electric Propulsion (SEP), with low thrust and very high $\mathrm{I}_{\mathrm{SP}}$, we only need to carry $850 \mathrm{~kg}$ of xenon. An example of an SEP that is well studied is the NEXT system. We are currently using NEXT as our baseline choice for retargeting maneuvers. 
The amount of fuel used must be traded against mission efficiency to come up with the optimal retargeting strategy. We are continuing to work out the trades between the obit the spacecraft are in, the starshade-telescope distance, the average slew angle, the time to do the slew, etc. It must be emphasized that during the time that the starshade is doing the retargeting maneuvers, the telescope will be conducting general astrophysics observations. So, the efficiency of the mission as a whole remains very high.

\section{B. Stationkeeping}

Once the starshade is positioned, it needs to remain aligned with the telescope for the instruments to take their measurements. The starshade needs to stay within the high-contrast shadow of the starshade, usually within a few meters of tolerance in the direction perpendicular to the starshade-telescope axis. The NWO system is very insensitive to motions along this axis; the tolerance in this direction is closer to \pm 100 $\mathrm{km}$. With a 4 meter telescope, and state of the art instrumentation, it would still take hours to days to obtain a high signal-to-noise detection of the spectrum from a planet, because it is so faint. Maintaining the alignment over this period of time requires us to carefully control both the position and velocity of the starshade.

We have used STK to model the forces that will act on the starshade as it tries to stay within a 5 meter box. We found that with a 50 meter starshade, solar torques account for about $30 \%$ of the total force, but the gravitational differential between the telescope and the starshade still dominates. For a given observation, the direction of the force is relatively constant within $5 \%$.

In principle, we can use the SEP system to counteract the perturbations on the starshade and maintain its alignment with the telescope. In practice, this faces two difficult problems. First, because there are constraints on the positioning of the starshade with respect to the sun, it is uncertain that we would be able to have full solar panel illumination during stationkeeping. To impose an additional restriction to maintain solar panel illumination would require reducing the available sky and would reduce the overall efficiency of the system. Another issue is that the thrust directions vary greatly with each observation, and are not necessarily lined up with the anti-solar direction. A SEP thruster has a realistic gimbaling angle of about $\pm 10^{\circ}$. Therefore, we would need at least 6 SEP thrusters to have full range of motion, firing thrusters in combination and taking the cosine loss to cover motions not in line with the thruster directions.

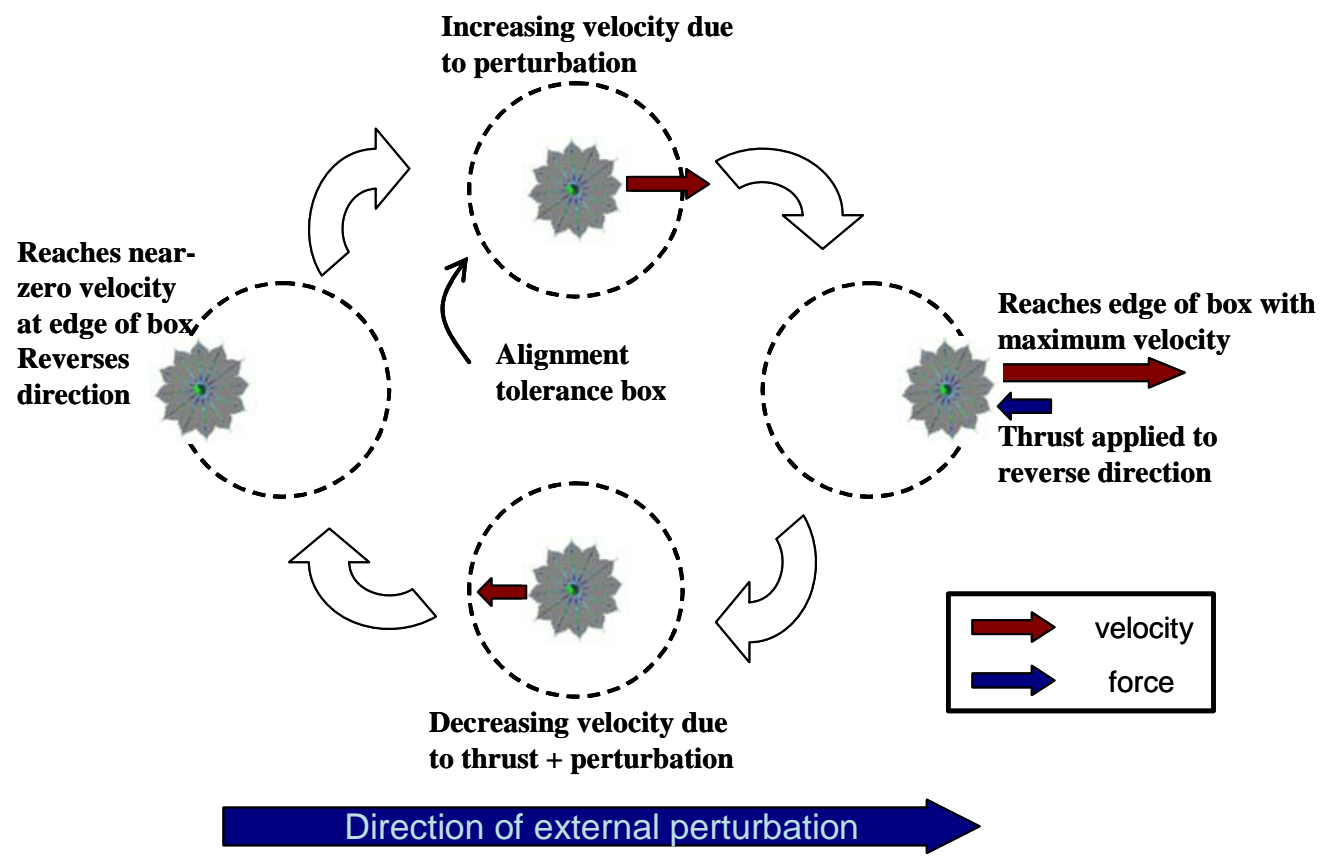

Figure 4. Maintaining the alignment of the starshade is done by firing the thrusters when the starshade reaches the edge of the tolerance box. The starshade then drifts back in the other direction until it has zero velocity at the other end of the control box. 
For these reasons, we have chosen a chemical propulsion system as our baseline for stationkeeping. In order to effectively utilize the high thrust of a chemical system, the stationkeeping operating scenario is as follows: the starshade starts with near zero velocity, relative to the telescope, and will drift according to the forces acting on it. When the starshade reaches the edge of the tolerance zone, we fire the thrusters in the opposite direction to the perturbing force. The starshade then drifts towards the center of the tolerance zone and reaches near-zero velocity at the opposite end of the tolerance zone. The perturbation once more pushes on the starshade, making it gain velocity and drift out of the tolerance zone. This scenario maximizes the time between thruster firings and therefore minimizes the number of times vibrations are excited on the starshade. Figure 4 shows a schematic of this stationkeeping scenario. Using our baseline orbit, our model indicates that the time between thruster firings ranges from 20 minutes to just over an hour.

During a 1 day observation, we estimate that $\sim 1 \mathrm{~m} / \mathrm{s}$ of $\Delta \mathrm{v}$ is required to maintain the alignment within a $\pm 1 \mathrm{~m}$ box. For the 130 observations during a 5 year mission, this means a total of $\sim 130 \mathrm{~m} / \mathrm{s}$ of $\Delta \mathrm{v}$. The total fuel used by the chemical propulsion system for $130 \mathrm{~m} / \mathrm{s}$ of $\Delta \mathrm{v}$ is $\sim 200 \mathrm{~kg}$, which is a very small fraction of the total starshade mass of $\sim 3000 \mathrm{~kg}$.

\section{Alignment Control}

Alignment control of NWO can be achieved through a variety of options. The fundamental requirements on the alignment control system come from comparing the shadow size generated by the starshade with the size of the telescope aperture. For example, a 50 meter starshade, operated at $80,000 \mathrm{~km}$ generates a shadow that has $10^{-10}$ contrast suppression within a 6 meter-diameter region. If this starshade is operated with a 2 meter telescope, the center of the shadow and the center of the telescope aperture must remain aligned to within \pm 2 meters. Working with a 4 meter telescope, this translates into an alignment requirement of \pm 1 meter.

For the purposes of these alignment controls, we assume that the starshade is performing the movements needed to keep the two spacecraft aligned. In almost all cases, it is preferable to move the starshade instead of the telescope, since the starshade is the lighter spacecraft.

In order for the starshade to arrive at the location where the center of the telescope is aligned with the center of the shadow, we must have knowledge of its position and be able to translate that knowledge into commands that move the starshade in the correct way. We have divided the trade space describing this process into three categories: coarse positioning, fine positioning, and on-target acquisition. We assume our baseline orbit is a halo orbit at L2.

Table 1

\begin{tabular}{|l|l|l|}
\hline Step & Method (Primary Instrument) & Accuracy \\
\hline $\begin{array}{l}\text { 1.Coarse } \\
\text { Positioning }\end{array}$ & Ground to Starshade & 3-D positioning within 50 - 100 km \\
\hline 2. Fine Positioning & $\begin{array}{l}\text { Starshade Aspect Camera looking } \\
\text { at telescope }\end{array}$ & $\begin{array}{l}\text { 1/30th pixel centroid: 20 mas accuracy } \\
\text { (within 2.5 - 6.5 m) }\end{array}$ \\
\hline $\begin{array}{l}\text { 3.On-target } \\
\text { Acquisition }\end{array}$ & Telescope primary optic & $\begin{array}{l}\text { Provides “on-target” signal } \\
\text { (within 1 m) }\end{array}$ \\
\hline
\end{tabular}

\section{A. Coarse Positioning}

Coarse positioning consists of placing the starshade in the general vicinity of the required location, defined to be within $100 \mathrm{~km}$ of the on-target position. As with all three steps, the coarse positioning trade space includes utilizing the ground, the starshade, or the telescope to direct the motion of the starshade. Coarse positioning needs to have wide-angle capability: in the event that the starshade loses tracking with the telescope they need to be able to search for each other.

With our baseline orbit at L2, we expect that a dedicated, ground-based network to be the optimal choice for coarse positioning. A ground-based network will have wide-angle capability and 3-D positioning accuracies of at least the required $50-100 \mathrm{~km}$. The trade space also includes options using a dedicated instrument on the starshade or the telescope. 


\section{B. Fine Positioning}

Fine positioning is defined as positioning the starshade to within a few meters of the on-target location. Since the ground is incapable of such resolution at L2, a dedicated instrument on the starshade or the telescope is necessary. There is a desired requirement to keep the telescope as free for general astrophysics as possible, and using a dedicated instrument on the telescope for this fine positioning phase means turning the telescope towards the starshade sooner than would be necessary otherwise. If a dedicated system needs to be installed, it is best installed on the starshade, which would be free to locate the telescope and perform the alignment with minimal interference to the telescope schedule.

Our current baseline is using an "aspect" camera mounted on the starshade. This camera is envisioned to have a $15 \mathrm{~cm}$ aperture and is based on the MAPs camera designed by the Naval Observatory ${ }^{3}$. It will look back at the telescope and direct the motion of the starshade until the telescope is correctly positioned against the field of stars behind it (the antipodal field). The field of view of the camera is on the order of a square degree which will make acquiring the telescope possible even when it starts out far from the correct position. By performing 1/30 pixel centroiding, we expect this camera to have the capability of aligning to within 6 meters of the on-target position.

\section{On-target Acquisition}

The final alignment of the system calls for very high-resolution observations of the location of the starshade. Clearly, this cannot be performed from the ground and is therefore up to an instrument on the starshade or the telescope. The precision required of the system for this process is high; it is unclear at this moment that there is a viable option other than using the main aperture of the telescope. It is possible, for example to use laser beacons to locate the starshade, but beamwidth divergence and signal strength over $80,000 \mathrm{~km}$ is a concern.

Using the main aperture of the telescope has several advantages, the primary being the size of the aperture. Using the largest aperture available allows us to obtain the highest-resolution data possible. Our baseline system is to use the focal plane of the telescope and the PSF of the star as it is blocked by the starshade. Figure 5 shows the telescope image-plane picture of the star as the starshade moves off axis up to 2 meters. The change is very pronounced, and can be detected within 0.5 meters of drift. We are continuing to work on the exact feedback loop, but this distortion can be used to sense when the starshade is out of alignment and in which direction.

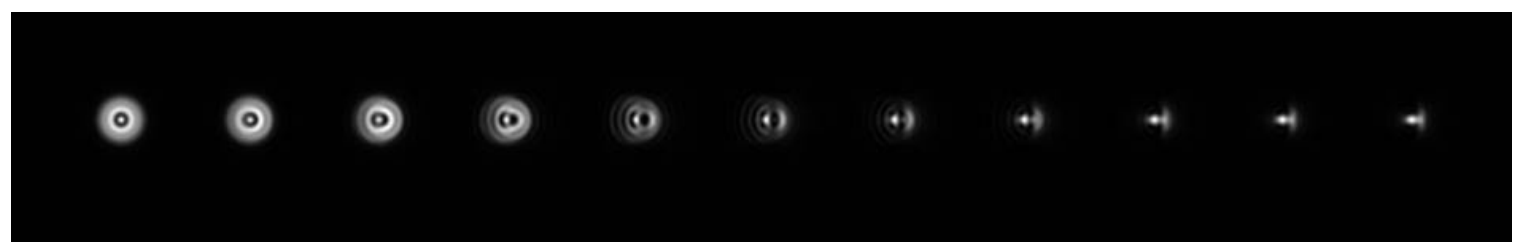

Figure 5. The shape of the stellar PSF is shown as the starshade drifts out of alignment with the telescope. The left-most picture shows the PSF of the star, as it appears in the telescope image plane, if the starshade and telescope are perfectly aligned. Subsequent images show the PSF as the starshade moves away from its on-axis position. The shape of the PSF distorts significantly as the starshade goes out of alignment. The rightmost image shows the PSF when the starshade is $\mathbf{2} \mathbf{m}$ from its nominal position. The shape of the PSF can be used to determine when the starshade is drifting out of alignment and correct it position.

Another, less well developed, possibility is to use reflections from the primary mirror into the field of view of the starshade aspect camera. The optimal system for on-target acquisition should have a large change in the signal between on-target and off-target and have high spatial resolution. This primary-mirror reflection, or "cat's eye" effect, falls off as the $4^{\text {th }}$ power of the offset of the starshade from its on-target position. This may therefore be a good choice and it will be studied further.

We have created a detailed model of how the active control loop would work using a test case. This case uses a JWST-heritage attitude control system on the starshade for courser alignment. This system consists of 4 reaction wheels and 2 star trackers. The fine positioning is done using the aspect camera on the starshade. A blending filter is used to combine the measurements from these two systems in order to determine the center of the control box. We conducted a test case where the mass of the starshade is 3000 $\mathrm{kg}$, the propulsion system has a thrust of $4.2 \mathrm{~N}$, and the position of the starshade is measured every $240 \mathrm{~ms}$. 
The requirement for alignment is that the starshade be within \pm 1 meter of its nominal position. We tested the case of the starshade starting out $1 \mathrm{~km}$ from its nominal position and moving into this box. The system successfully moved the starshade into the box in $\sim 3$ hours using $4 \mathrm{~kg}$ of fuel. Further refinements on the initial conditions and movement algorithms will be able to reduce the acquisition time and fuel. We are confident that we will be able to develop an optimized solution for alignment control.

Table 2

\begin{tabular}{|l|l|l|l|}
\hline \multicolumn{1}{|c|}{ Link } & \multicolumn{1}{c|}{ Data Rate } & Frequency & \multicolumn{1}{c|}{ Data } \\
\hline 1. Ground to Telescope (Omni) & $1-4 \mathrm{kbps}$ & S band & Command/ Ranging \\
\hline 2. Telescope to Ground (Omni) & $0.125-2 \mathrm{kbps}$ & $\mathrm{S}$ band & Telemetry/ Ranging \\
\hline 3. Ground to Telescope (MGA) & $1-16 \mathrm{kbps}$ & $\mathrm{S}$ band & Command/ Ranging \\
\hline 4. Telescope to Ground (MGA) & $1-10 \mathrm{kbps}$ & $\mathrm{S}$ band & Telemetry/ Ranging \\
\hline 5. Telescope to Ground & $28 \mathrm{Mbps}$ & Ka band & Science/ Telemetry \\
\hline 6. Telescope to Starshade & $4 \mathrm{kbps}$ & $\mathrm{S}$ band & Command/ Ranging \\
\hline 7. Starshade to Telescope & $150 \mathrm{kbps}$ & $\mathrm{S}$ band & Telemetry + Science \\
\hline 8. Ground to Starshade & $1-16 \mathrm{kbps}$ & $\mathrm{S}$ band & Command/ Ranging \\
\hline 9. Starshade to Ground & $0.125-2 \mathrm{kbps}$ & $\mathrm{S}$ band & Telemetry/ Ranging \\
\hline
\end{tabular}

\section{Communications design}

The NWO architecture creates special requirements for the communication system. The starshade and the telescope need to communicate with each other for alignment, the telescope needs to communicate with the ground for science data return, and, while the starshade can use the telescope as a relay, in order to reduce risk the starshade should be able to communicate with the ground.

For our baseline system, science data is returned via a Ka-band link that is capable of providing a 28 Mbps data stream. Command and ranging for all links are all performed in the S band. There are both Sband omni and S-band high-gain antennae on the starshade and the telescope. The high-gain antennae are designed for command ranging for alignment and possible science data return to and from the telescope. The omni's are backup systems that are capable of lower bit-rate communication between the starshade and the ground and between the starshade and the telescope. The linkage and rates are shown in the Table 2.

\section{Conclusion}

The NWO mission involves two widely-separated spacecraft which must operate together to observe planets around nearby stars. Operating these spacecraft involves issues of orbits, scheduling, propulsion, etc. We have explored the trade space for each of these systems and chosen a baseline that fulfills all of the program requirements.

We have chosen a halo orbit around L2 as the baseline orbit. It provides for large sky coverage, good communication, and reasonable fuel costs to get into position and conduct the mission.

To retarget the starshade from one star to the next, a solar electric propulsion system provides the best fuel efficiency. We have shown that we can visit a reasonable number of targets using this system. In order to keep the starshade aligned with the telescope, we will use a chemical propulsion system.

During an observation, the starshade must remain aligned with the telescope to within about \pm 1 meter. We have developed a control algorithm that can move the starshade from its previous position into alignment with the telescope and the next target star. We currently see this as involving three steps: coarse alignment using ground-based data, fine alignment using an aspect camera mounted on the starshade, and on-target acquisition using the aperture of the telescope itself.

For all of the systems we have explored here, the trade space remains open. We have shown that a solution exists for all subsystems that allows the NWO mission to operate as required. However, these may not be the optimized solutions. Details of the optimal operating scenarios are still being worked out and many of the systems we outlined here might change. We have shown that NWO is a viable mission and that the operations can be conducted in such a way as to meet all the mission requirements. 


\section{References}

${ }^{1}$ Cash W., "Detection of Earth-like planets around nearby stars using a petal-shaped starshade”, Nature, Vol. 442, No. 7098, 2006, pp.51-53

${ }^{2}$ Polidan, R., "Future Mission Architecture and Technology Roadmaps", UV/Optical/IR Space Telescopes: Innovative Technologies and Concepts III, Edited by H.A. MacEwen and J.B. Breckinridge, Vol. 6687, SPIE, 2007, (to be published)

${ }^{3}$ Gaume, R., Dorland, B., Makarov, V., Zacharias, N., Johnston, K., and Hennessy, G., "Space Astrometry With The Milli-Arcsecond Pathfinder Survey (MAPS): Mission Overview And Science Possibilities”, Nomenclature, Precession and New Models in Fundamental Astronomy, Joint Discussion 16, 26th meeting of the IAU, p. 26 